\title{
Packing Fractions and Maximum Angles of Stability of Granular Materials
}

\author{
J. Olson, M. Priester, J. Luo, S. Chopra and R.J. Zieve \\ Physics Department, University of California at Davis
}

\begin{abstract}
In two-dimensional rotating drum experiments, we find two separate influences of the packing fraction of a granular heap on its stability. For a fixed grain shape, the stability increases with packing fraction. However, in determining the relative stability of different grain shapes, those with the lowest average packing fractions tend to form the most stable heaps. We also show that only the configuration close to the surface of the pile figures prominently.
\end{abstract}

Granular materials are studied in materials science, geology, physics, and engineering [1]. Their unusual dynamical behavior has attracted much recent attention [2, 3, 4], and even the more staid static issues generate steady interest [5, 6]. One question is how much of granular behavior can be derived from purely geometrical considerations. In practical materials, particle deformation and surface effects such as cohesion and agglomeration also play major roles, masking the influence of geometry. Furthermore, studying grain shape is a difficult endeavor. The experimental challenge is in creating uniform but non-spherical shapes. Recent efforts include twodimensional studies of regular pentagons [7, 8] and a threedimensional experiment using M\&M's [9]. On the theoretical side, the problem is how to treat the interaction of grains as they move past each other.

Work linking geometry to behaviors other than packing is yet more scarce. One rare attempt involves predicting avalanches in a granular pile [10], a question at the border between statics and dynamics. Granular piles, such as sandpiles, exhibit characteristic angles for their free surfaces. One is the repose angle, below which the pile is stable. If the surface becomes steeper than the repose angle, for example if the pile is tilted or if new grains are added, then the heap may undergo an avalanche in which grains all along the slope move, resulting in a lower angle. In practice, avalanches do not begin until the angle exceeds what is known as the maximum angle of stability, which is typically several degrees larger than the repose angle. Once started, an avalanche continues until the pile surface is again less steep than the repose angle. Albert et al. [10] derive a maximum angle of stability from local geometry, beginning with a regular tetrahedron of spheres. In addition to safety issues, understanding avalanches is important in fields such as geology and soil mechanics, where granular matter can flow along inclined surfaces.

Both theoretical and experimental work relate the packing fraction of a heap to its stability, for the special case of spherical grains [11, 12, 13]. The experiments involve packing spheres under pressure to achieve different initial packing fractions, then tilting the heap and noting the angle of the first avalanche. As the spheres are packed more tightly, the maximum angle of stability increases. In both these measurements, the packing fraction is known only for the initial, artificially constructed arrangement. They do not test whether packing fraction also affects stability in the configurations that actually occur after an avalanche. One set of these experiments [11] uses not only smooth spheres but also "rough" spheres and angular grains. The two very different non-spherical shapes sustain similar maximum angles, higher than that of smooth spheres.

Here we revisit the role of density, extending our study to naturally occurring configurations and to non-spherical grains. We work in two dimensions, which makes visualizing an entire configuration much easier than for a three dimensional heap. Our grains are composed of spherical ball bearings, welded together in clusters of up to 9 balls. The balls in each cluster are part of a two-dimensional triangular lattice. Working with sphere clusters has various advantages. The maximum possible density is always that of a triangular lattice of the component spheres. Spheres minimize friction and blocking effects as the shapes move past each other. Finally, our system lends itself to comparison with computer experiments, since checking for overlaps, a challenging part of typical simulations, is trivial for sphere clusters.

We find that packing fraction indicates pile stability for both spherical and non-spherical shapes, but with a twist. When comparing piles composed of different grains, low average packing fraction indicates stability. After presenting our experimental results, we offer an explanation for this behavior and other observations about the influence of grain shape.

As described elsewhere [14], we weld together $\frac{1}{8}$-inch diameter carbon steel ball bearings to make dimers; trimers (three balls in a straight line); triangles of three or six balls; diamonds of four, six, or nine balls; trapezoids of five or seven balls; and hexagons of seven balls. The left column of Table illustrates these shapes.

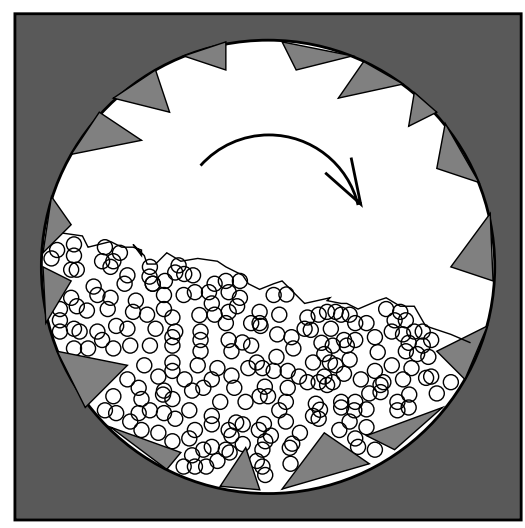

FIG. 1: Tumbler for two-dimensional granular heap. The triangles transform the container boundary from a circle to an irregular shape.

Our tumbler, shown schematically in Figure 10 is a sheet of 
aluminum, $\frac{1}{8}$ " thick with a circular hole 14 inches in diameter cut from its center. The aluminum is sandwiched between two $\frac{1}{2}$ " thick sheets of Plexiglas, which constrain the balls to move in a single layer. The plane of the tumbler is vertical. A central axle attaches the tumbler to a stepper motor which controls the rotation rate. For all measurements here the rotation rate is about $500 \mu \mathrm{Hz}$, or one full turn in about thirty minutes. We use this slow speed so that the rotation of the container itself during an avalanche remains negligible, and the avalanches are discrete events. To prevent the balls from sliding along the wall of the container during rotation, a thin strip of rubber is glued to the inner edge of the aluminum. In some measurements, as described below, an irregular boundary was created by attaching aluminum triangles along the edge of the hole.

For each shape we use a total mass of $365.6 \pm 0.3 \mathrm{~g}$, so that the grains fill a similar portion of the tumbler. We rotate the tumbler for about 30 minutes, which generally yields 20 to 30 discrete avalanches. The entire rotation is recorded with a digital video camera. Afterwards the avalanches are identified by eye, and the video frames immediately before and after each avalanche are uploaded to a computer.

To simplify the image processing, we use a solid red background inside the tumbler and solid white around the outside. These colors allow a computer program to identify easily the region occupied by the heap. Since we know both the total mass of the grains and the mass per grain, we can convert the area of the heap to a packing fraction. We also fit a line to the free surface of the heap and use it to define the angle from horizontal of the surface. In this way we extract the angle and packing fraction for each image.

On removing the shapes from the tumbler after a measurement, we sort and count any broken grains. The maximum angle of stability is sensitive to broken shapes; so if over $10 \%$ of the original pieces break during a measurement, we discard the data and run that shape again. In most cases breakage is less than $3 \%$ of the original shapes.

The black bars of Figure 2 show the average angle just before an avalanche for several different shapes. Standard errors are typically one degree, and never more than 1.5 degrees, so the variations among shapes are significant. Two shapes, diamonds and hexagons, form significantly more stable heaps than the other shapes.

These angles are very different from the results of our previous work in a rectangular container, which allows a single crystalline region [15]. To test that boundary effects do not dominate the variations we find among shapes, we inserted 16 triangles in an irregular pattern along the surface of the circle and repeated the measurements. The results, shown as red bars in Figure 2, are qualitatively the same. Diamonds and hexagons remain much more stable than the other shapes.

The quantitative differences between the containers could be an effect of the boundary surface. Another contribution may come from small differences in the numbers of broken shapes in the two sets of measurements.

In the irregular container, we extended the measurements to several additional shapes. All data from this setup are shown in Table I] There is no clear pattern of how geometry affects critical angle, beyond the general observation that larger

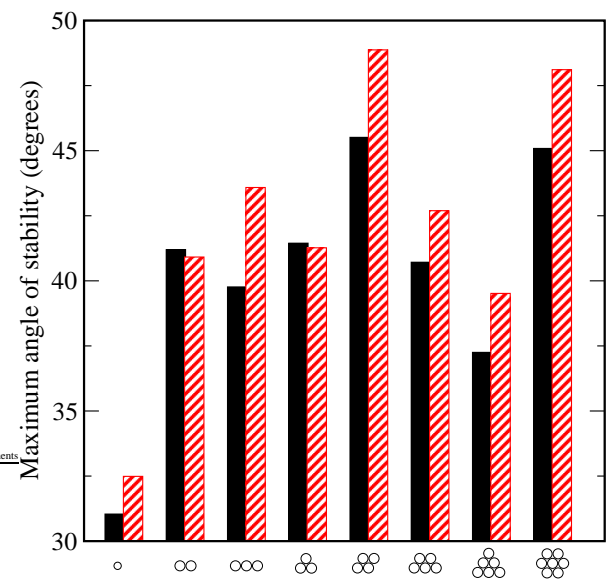

FIG. 2: Average angle for avalanche onset for eight different shapes. Black: container with circular boundary. Red: container with irregular boundary. The shapes used are indicated along the horizontal axis, arranged in order of size.

shapes support higher angles. Given the scalloped boundaries of our shapes, which allow some interlocking, this seems natural.

TABLE I: Average maximum angle of stability $\left(\theta_{m}\right)$ and repose angle $\left(\theta_{r}\right)$ for eleven shapes in a container with irregular boundary. Standard errors are also given. Shapes are ordered from smallest to largest.

\begin{tabular}{|c|c|c|c|c|}
\hline & $\theta_{m}$ & $\sigma\left(\theta_{m}\right)$ & $\theta_{r}$ & $\sigma\left(\theta_{r}\right)$ \\
\hline - & 33.8 & 0.7 & 26.3 & 0.6 \\
\hline$\bullet \bullet$ & 42.0 & 0.6 & 32.9 & 0.5 \\
\hline$\bullet \bullet$ & 43.8 & 1.0 & 34.5 & 0.8 \\
\hline$\therefore$ & 41.2 & 0.5 & 31.3 & 0.3 \\
\hline$\because \because$ & 49.1 & 1.0 & 38.2 & 0.9 \\
\hline$\therefore$ & 44.9 & 0.8 & 33.6 & 0.7 \\
\hline$\therefore$ & 39.5 & 0.4 & 29.3 & 0.5 \\
\hline$\because \because$ & 51.8 & 1.0 & 37.8 & 0.9 \\
\hline$\because \bullet$ & 48.7 & 1.3 & 35.0 & 1.0 \\
\hline$\therefore \because 8$ & 50.6 & 1.1 & 38.7 & 0.7 \\
\hline$\because \because{ }^{\circ}$ & 50.9 & 1.5 & 36.6 & 0.9 \\
\hline
\end{tabular}

We also characterize each configuration by its packing fraction. Figure 3 shows packing fraction and maximum angle attained for each avalanche with the small triangles. As in the earlier work with compacted media in three dimensions [11, 12, 13], the two properties are positively correlated. This also agrees with the observation that interlocking and jamming, which can increase the stability angle, occur more easily at higher packing fractions [15]. Strikingly, eight of the ten other shapes exhibit a similar correlation between the packing fraction and the angle of the ensuing avalanche, with correlation coefficients between 0.4 and 0.71 . (The remaining shapes, the large triangles and large trapezoids, show no significant correlation between the two.) Thus we confirm experimentally that a connection between pile density and max- 
imum angle of stability exists for a variety of shapes, although it may not be universal.

Even more importantly, our measurements relate these properties for naturally occurring configurations rather than for the artificially compacted initial arrangements of the previous work. Finding similar results for packings created so differently and in both two and three dimensions raises the hope of a general principle applying to a wide variety of configurations. Furthermore, in many practical situations, a pile is in fact formed from previous avalanches. Our work also highlights the importance of packing fraction in determining stability, since we detect effects of pile density despite its much smaller variation for our samples than in the compressed piles.

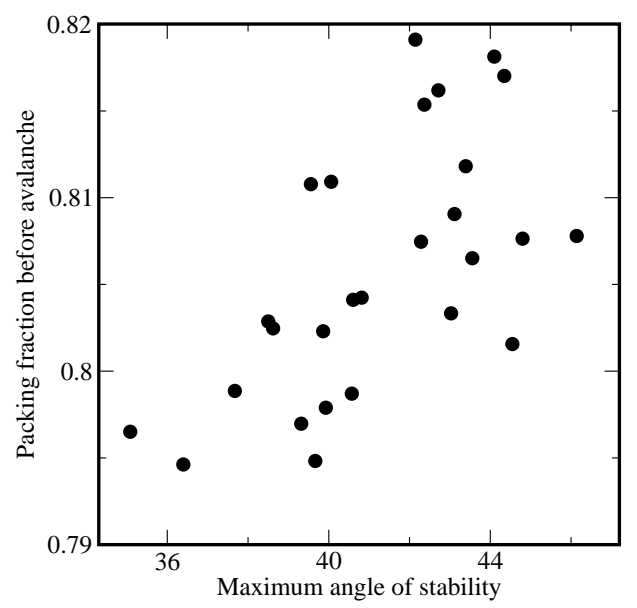

FIG. 3: Packing fraction and critical angle for the individual avalanches of the small triangle shapes ( 3 balls). The correlation coefficient is 0.626 .

In addition to connecting pile density and stability angle for the individual avalanches of each shape, we compare the behavior among shapes. Here a far less intuitive influence of packing fraction appears. Figure 4 again plots packing fraction against maximum angle, but here each point represents an average over all avalanches for a single shape. Now the correlation is actually in the opposite direction from that of Figure 3 high packing fractions tend to yield low critical angles.

The apparently opposite influence of packing fraction within a shape and across different shapes may be understood by considering how the pile is formed. From our measurements on individual shapes, we know that tightly packed configurations are more stable. During an avalanche, a necessary condition for flow to cease is a stable instantaneous arrangement of grains. Furthermore, grain motion always requires expansion so that the particles have room to move [16]. As the avalanches stops, the packing fraction again increases. Thus the moving grains pass through configurations with a range of packing fractions. Many of the arrangements with the lowest packing fractions are unstable, or at least not stable enough to absorb the momentum of the moving grains. As the packing fraction increases, the arrangements are more likely to be stable and allow the avalanche to stop. If a shape has a low average packing fraction, its avalanches must stop relatively early, and the shape is likely to support a wide range of stable

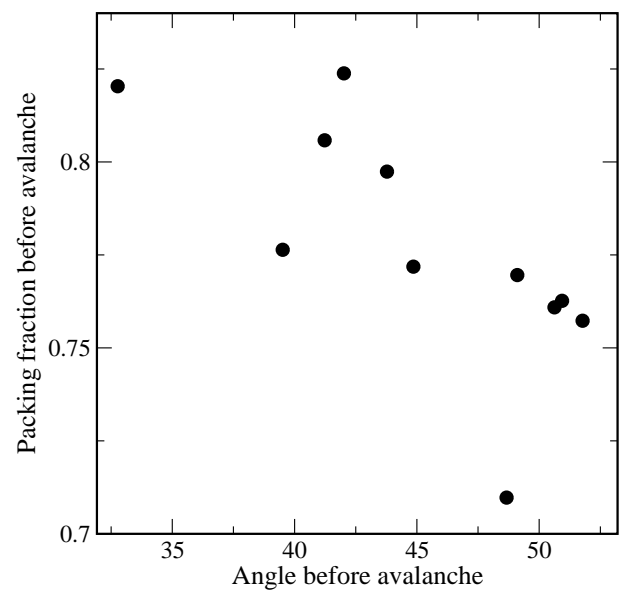

FIG. 4: Average packing fraction and critical angle for the eleven different shapes. Each filled circle represents one shape.

configurations.

Rotation is another means of sampling different grain arrangements, since the stability of any configuration depends on the direction of gravity. If more arrangements than usual are stable for a particular grain shape, then the new configurations reached by tilting a heap of such grains are more likely to be stable. On average the heap will take longer to reach an unstable configuration and trigger an avalanche. The negative correlation we observe between packing fraction and maximum angle of stability comes about because low-density configurations are most likely to occur with shapes of generally high stability. This logic implies that particularly high critical angles should be found for tightly packed grains with a low random loose packing value.

TABLE II: Correlations among packing fractions before $\left(\rho_{i}\right)$ and after $\left(\rho_{f}\right)$ an avalanche, maximum angle of stability $\left(\theta_{m}\right)$, angle of repose $\left(\theta_{r}\right)$, and change in angle during an avalanche $(\Delta \theta)$. Values are the averages of the correlation coefficients calculated for all 11 shapes.

\begin{tabular}{l|rrrr} 
& $\rho_{i}$ & $\theta_{r}$ & $\rho_{f}$ & $\Delta \theta$ \\
\hline$\theta_{m}$ & 0.475 & -0.269 & 0.032 & 0.841 \\
$\rho_{i}$ & & -0.105 & 0.613 & 0.399 \\
$\theta_{r}$ & & & 0.290 & -0.728 \\
$\rho_{f}$ & & & & -0.138
\end{tabular}

Monitoring successive avalanches allows us to study other correlations among the configurations as well. Table 1 deals with five variables: packing fraction and angle before and after each avalanche, and the change in angle. The table shows the correlation between each pair of quantities, averaged over all eleven shapes. There is no significant correlation between initial packing fraction and repose angle, or between final packing fraction and maximum angle of stability. We also find directly that the angles of successive avalanches are uncorrelated. Previous experiments found a similar lack of correlation in the size of successive avalanches [17], suggesting that a single avalanche completely resets the system memory. 
On the other hand, the packing fractions of successive avalanches (or equivalently, the packing fractions before and after a single avalanche) are highly correlated. This is hardly surprising, given that any single avalanche leaves about half of the ball bearings completely unaffected. In addition to the stationary regions, there are typically several large clumps within the pile that rotate slightly during the avalanche but have not change in their internal arrangements. With this in mind, the correlation is if anything lower than expected. The implication is that the density varies more strongly in the top few layers, which reconfigure completely during an avalanche, than in the rest of the heap.

Combining these observations, we see that significant packing fraction correlations between consecutive avalanches arise from the stationary lower layers, but that successive avalanches show no correlation in stability angle. Consequently, the correlation between packing fraction and maximum angle of stability must depend only on the packing fraction in the upper, mobile layers. The heap density within these layers, which we cannot easily calculate, would likely show a much stronger relationship with the stability angle than the overall density as in Figure 3

Finally, there is a small but consistent negative correlation between critical angle and repose angle. This may happen because more momentum builds up during an avalanche that begins on a steep slope, enabling the avalanche to continue longer. The one exception is the single balls, which have positive correlation between critical and repose angles as well as much weaker connections between avalanche size and the initial and final angles.

In conclusion, we show that packing fraction plays a dual role in predicting the stability of a two-dimensional heap. For a given grain shape, denser packings are generally more stable; and we have extended this result beyond the previous measurements on artificially packed spheres in three dimensions. However, when comparing different shapes, those with the lowest packing fractions have the highest maximum stability angles on average. We also find that only the packing fraction of the top few layers of the heap figures strongly affects stability, so the correlations would likely be much cleaner if a packing fraction calculation could be confined to this region.

It would be interesting to test how widely the inverse correlation between maximum stability angle and packing fraction holds. The shapes we used are all clusters of spheres, confined to two dimensions. Experiments or simulations could also be done on shapes such as ellipses or polygons, and extended to three dimensions.

We are also expanding our work to heaps containing a mixture of grain shapes, which drastically enlarges the phase space for measurements. In the course of the present work, we observed that a small fraction of broken shapes can significantly change the critical angle. We expect tests of shape mixtures to help explain why.

We thank C. Olson and C. Reichhardt for discussions. This work was supported by the National Science Foundation under DMR-9733898.
[1] J. Duran, Sands, Powders, and Grains: An Introduction to the Physics of Granular Materials (Springer-Verlag, New York, 2000).

[2] A. Kudrolli, "Size separation in vibrated granular matter," Rep. Prog. Phys., 67, 209 (2004).

[3] J. Rajchenbach, "Granular flows," Adv. Phys. 49, 229 (2000).

[4] R.P. Behringer, "The scientist in the sandbox: time-dependence, fractals and waves," Int. J. Bifurcation Chaos Appl. Sci. Eng. 7, 963 (1997).

[5] S.F. Edwards and D.V. Grinev, "Granular materials: towards the statistical mechanics of jammed configurations," Adv. Phys., 51, 1669 (2002).

[6] D. Bideau and A. Hansen, Disorder and Granular Media (North-Holland, Amsterdam, 1993).

[7] F. Cantelaube, Y. Limon-Duparcmeur, D. Bideau, and G.H. Ristow, "Geometric analysis of avalanches in a 2D drum," J. Phys. (France) I 5, 581 (1995).

[8] Y. Limon Duparcmeur, A. Gervois, and J.P. Troadec, "Crystallization of Pentagon Packings," J. Phys.: Condens. Matter 7, 3421 (1995).

[9] A. Donev, I. Cisse, D. Sachs, E.A. Variano, F.H. Stillinger, R. Connelly, S. Torquato, and P.M. Chaikin, "Improving the density of jammed disordered packings using ellipsoids," Science 303, 990 (2004).
[10] R. Albert, I. Albert, D. Hornbaker, P. Schiffer, and A.L. Barabasi, "Maximum angle of stability in wet and dry spherical granular media," Phys. Rev. E 56, R6271 (1997).

[11] R.L. Brown and J.C. Richards, Principles of Powder Mechanics (Pergamon, Oxford, 1966).

[12] J.R.L. Allen, "The angle of initial yield of haphazard assemblages of equal spheres in bulk," Geologie en Mijnbouw 49, 13 (1970).

[13] P. Evesque, D. Fargeix, P. Habib, M.P. Luong, and P. Porion, "Pile density is a control parameter of sand avalanches," Phys. Rev. E 47, 2326 (1993).

[14] I.C. Rankenburg and R.J. Zieve, "Influence of shape on ordering of granular systems in two dimensions," Phys. Rev. E63, 1303 (2001).

[15] C.J. Olson, C. Reichhardt, M. McCloskey, and R.J. Zieve, "Effect of grain anisotropy on ordering, stability, and dynamics in granular systems," Europhys. Lett. 57, 904 (2002).

[16] O. Reynolds, "On the dilatancy of media composed of rigid particles in contact," Phil. Mag 20, 469 (1885).

[17] P. Evesque, "Analysis of the statistics of sandpile avalanches using soil-mechanics results and concepts," Phys. Rev. A 43, 2720 (1991). 